\title{
A first principles thermal losses model of the TCP-100 parabolic trough collector based on the Modelica Standard Library
}

\author{
Julia Pérez ${ }^{1}$ Luis J. Yebra ${ }^{1}$ Francisco M. Márquez ${ }^{2,3}$ Pedro J. Zufiria ${ }^{3}$ \\ ${ }^{1}$ Plataforma Solar de Almería, CIEMAT, 04200 Tabernas, Spain \\ perezruizjulia@gmail.com, luis.yebra@psa.es \\ ${ }^{2}$ Dpto. de Automática, Universidad de Alcalá (UAH), 28801 Alcalá de Henares, Spain, \\ francisco.marquez@uah.es \\ ${ }^{3}$ Dpto. Matemática aplicada a las TIC, Information Processing and Telecommunications Center, ETSI \\ Telecomunicación, Universidad Politécnica de Madrid (UPM), 28040 Madrid, Spain, \\ francisco.mgarciadalumnos.upm.es, pedro.zufiria@upm.es
}

\begin{abstract}
The TCP-100 parabolic trough collectors (PTC) research facility at Plataforma Solar de Almería (CIEMAT) has been specially designed for the development of research activities in Automatic Control of PTC solar thermal power plants. The development of advanced control techniques for this kind of facilities requires dynamic models that should be successfully used in advanced controllers. An important part of these models is the thermal losses submodel, that traditionally has been considered as an experimental steady state correlation. In this paper, a work in progress about a first principles based model of the losses to the environment of a parabolic trough collector is presented, based on the physical phenomena inside any parabolic trough collector during the operation of the TCP-100 solar field. Concerning this model, the main contribution of the paper is to include the dependence of the air velocity close to the PTC. The implementation of the model in the Modelica language has been done prioritizing the use of the Modelica Standard Library classes. Some simulations results of this model with theoretical parameters values under typical operating conditions of the TCP-100 plant are presented, showing higher losses to the environment when compared with the information provided by the TCP-100 manufacturer.
\end{abstract}

Keywords: parabolic trough collector, thermal losses, object oriented modelling, Modelica Standard Library

\section{Introduction}

The main research facility at Plataforma Solar de Almería (PSA, www.psa.es) to test models and control algorithms has been the ACUREX parabolic trough collector (PTC) plant throughout its 32 years of life. Modelling and Control has been a research line at PSA under which mathematical models and advanced control techniques have been designed, implemented and tested. The ACUREX was replaced by the TCP-100 facility, shown in Figure 1, that presents important structural differences with respect to its predecessor, as outlined in section 2.

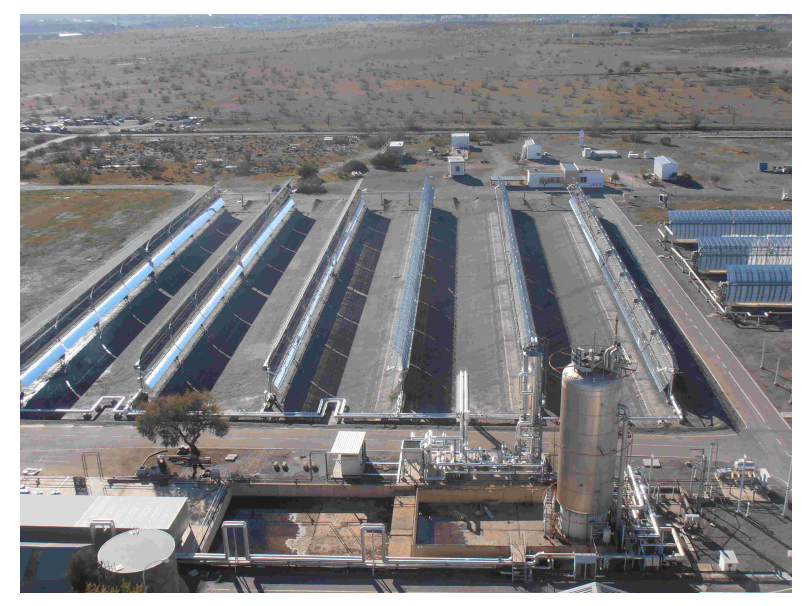

Figure 1. The TCP100 PTC solar research facility at Plataforma Solar de Almería (PSA-CIEMAT), (Pérez et al. 2018).

So far, modelling works based on the TCP-100 facility are: (Gallego, Yebra, Camacho, and Sánchez 2016), where a non-linear distributed parameter model of the TCP-100 solar field is presented; and (Pérez et al. 2018) in which the authors present a first principles system level dynamic model based on the project engineering data of the plant, complemented with several simulation experiments covering several design operation modes. Regarding control research activities, the only currently published work is (Gallego, Yebra, and Camacho 2018) where a Gain Scheduling Model Predictive Controller based on the model in (Gallego, Yebra, Camacho, and Sánchez 2016) is presented. In (Yebra, Márquez, and Zufiria 2020) a hybrid model of the TCP-100 facility is presented, covering the different operation modes of the plant and proving its applicability for operation training purposes. This hybrid model is implemented using both the StateGraph formalism in the Modelica object-oriented modelling language, and the Dymola tool. Currently, port-Hamiltonian modeling of multiphysics systems and object-oriented implementation techniques (Márquez, Zufiria, and Yebra 2020) are being applied to the modelling of the TCP-100 
facility.

Usually, the losses model of a PTC has been approximated by a static nonlinear correlation-based model depending on the environment variables. Although the losses model presented here is more detailed than the other models used for control purposes, it still has low computational requirements, this fact allowing an efficient use of the model when it is connected to the other parts of the collector model. This model could be employed for the estimation of the losses and diagnosis based on its predictions independently in real-time applications during the operation of the plant, even in solar plants with a large number of collectors. We will test this model in the TCP-100 research plant to estimate each PTC power lost in real time.

The remainder of this article is organized as follows. In section 2 an introduction of the TCP-100 facility is presented, including the PTCs to which the model applies. In section 3 the model is presented, and in section 4 the implementation of the model with the Modelica Standard Library (MSL) is outlined. In section 5 different simulation results of the model are presented; finally, some conclusions are drawn in section 6 .

\section{The TCP-100 research plant}

The plant is composed of two thermofluid circuits with different heat transfer fluids (HTFs) in each of them: Syltherm 800 in the solar field (primary circuit) and Therminol 55 in the storage part (secondary circuit). Both circuits are connected thermally by a heat exchanger (HEX). Figure 1 shows the complete facility where both parts (solar field and storage) are shown. In comparison to the ACUREX field, there are structural changes concerning the tanks system: there is now a new buffer tank T-2 in the primary circuit of $10 \mathrm{~m}^{3}$ before the solar field pump. The previous tank T- 1 of $115 \mathrm{~m}^{3}$ has been maintained in the storage part (secondary circuit). The speed of the pumps for each of the two circuits can be controlled by the internal control loops inside variable frequency drives (VFD) connected to both pumps. An air cooler in the secondary circuit with a VFD has been installed to vary the cooling power in the storage part (the ACUREX field did not allow this). These are some of the new and significant structural changes of the solar thermal facility, that will require the application of more sophisticated operation modes. A diagram of the plant is shown in Figure 2.

\subsection{The TCP-100 primary circuit}

The TCP-100 primary circuit is mainly constituted by the solar field that occupies most of the plot, as it can be seen in Figure 1. The solar field is composed of three loops, each one with two PTCs in a North-South orientation. Each PTC is $100 \mathrm{~m}$ long, and it is formed by 8 modules connected in series. Figure 3 shows the first PTC of the first loop, numbered from the inlet (and coolest part) of the loop.

The PTCs in each loop are connected in the South extreme, and the coldest PTC will be always the first in the

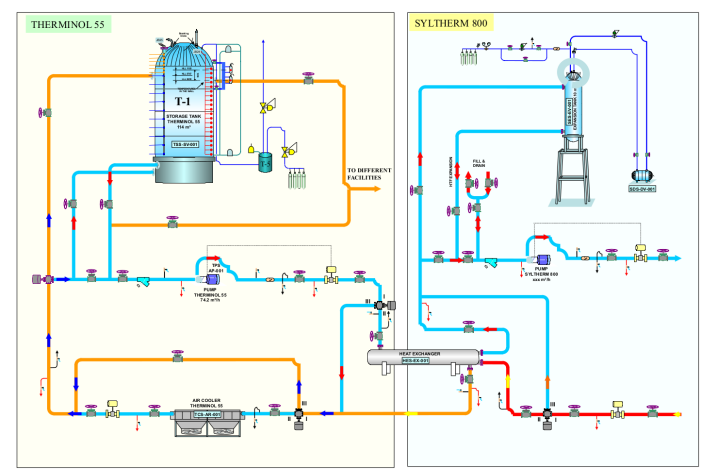

Figure 2. Diagram of the TCP-100 plant showing the main components in both subcircuits: primary (Syltherm800) and secondary (Therminol 55).

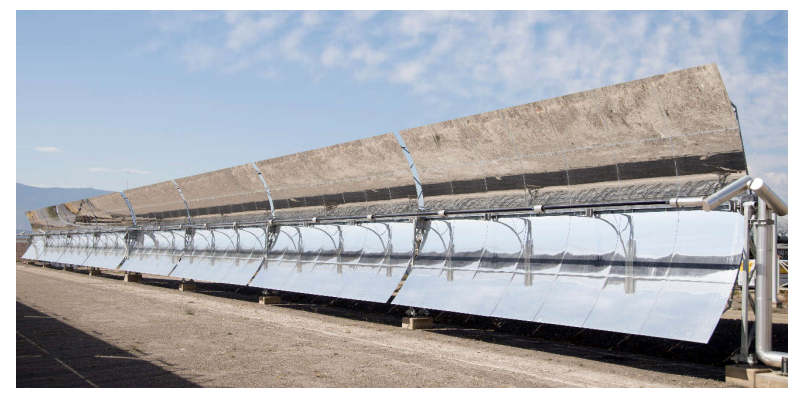

Figure 3. First PTC of the first loop of the TCP-100 solar field, (Pérez et al. 2018).

row, placed at the right part of each loop in Figure 1. Each of them has two PTCs connected in series, ordered from 1 (rightmost) to 6 (leftmost). The first loop is formed by the connected pair 1st-2nd (right loop), the second one by 3rd4th (center loop) and the third one by 5th-6th (left loop).

The thermal losses (sub)model of a PTC plays an important role in any solar thermal plant model. It will be applied to all of the PTCs in any solar thermal facility. In our experimental facility TCP-100 we have 6 PTC units but an industrial plant could contain thousands of them. This model has an important influence on the efficiency of the plant operation and has a marked importance in the design of advanced control strategies. This fact has motivated our work on deriving an independent submodel based on first principles for the thermal losses in a PTC, that should be directly connected to other model parts of the collector; or it could also be used independently as a standalone model.

The technology of a parabolic trough collector is described with detail in (Zarza 2003), where the components involved in the losses to the environment are presented.

\section{TCP-100 parabolic trough collector thermal losses model}

The model that describes the heat losses is founded in well known physical phenomena: conduction, convection and radiation (Bejan 2016). With the objective of using this model independently of the other dynamics in a PTC, we 
will consider boundary conditions the temperature of the HTF inside the PTC absorber pipe, the ambient temperature $T_{a m b}$, and the wind velocity close to the PTC absorber pipe. Although the solar irradiance is usually an important boundary condition in solar thermal models, it is not considered in this case since it has no direct implication in the presented losses model. The presented model is a first principles model, including correlations to approximate the solution of the heat transfer coefficients. The equations of conservation and the energy flows are implemented in the MSL classes.

Figure 4 shows a simplified representation of the heat flows in a PTC absorber pipe. In such figure, $\dot{Q}_{\text {incident }}$ and $\dot{Q}_{\text {lost }}$ represent, respectively, the heat flow rates incident into and lost away from the absorber pipe.

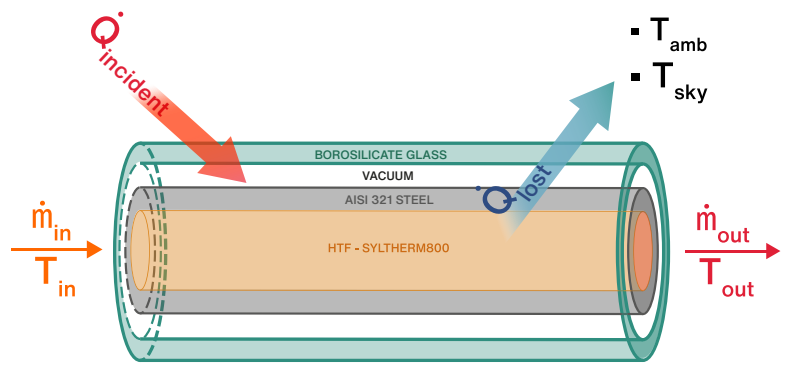

Figure 4. Heat flows in a PTC.

In Figure 5 a representation of the internal heat flow rates between the different sections is sketched.

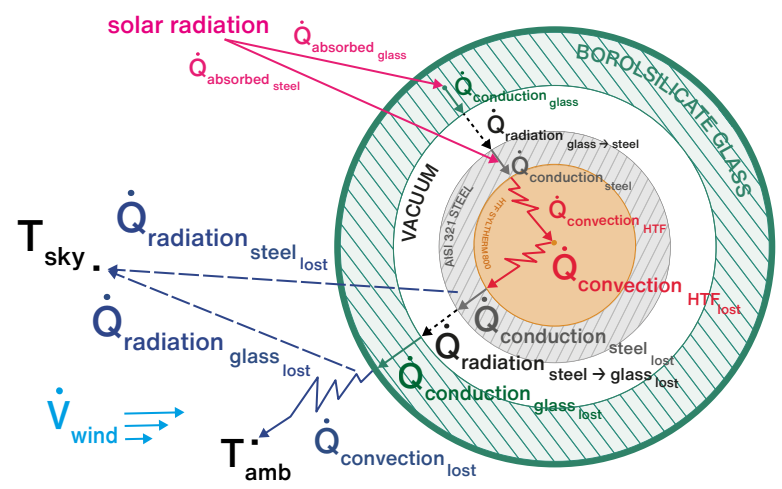

Figure 5. Heat flows in a section of a PTC.

The equations for the thermal losses model are detailed in (Wagner and Gilman 2011), (Pérez et al. 2018) and (Zarza 2003), and the flows have been organized for the implementation in the MSL as follows:

- $\dot{Q}_{a b s o r b e d, g l a s s}$ is the heat flow absorbed by the envelope glass from the reflected concentrated solar radiation.

- $\dot{Q}_{a b s o r b e d, s t e e l}$ is the heat flow that passed through the envelope glass and is absorbed in the pipe (steel).

- $\dot{Q}_{\text {conduction,glass }}$ is the conduction heat flow inside the glass envelope.
- $\dot{Q}_{\text {radiation,glass-steel }}$ is the radiation heat flow between the glass envelope and the pipe.

- $\dot{Q}_{\text {conduction,steel }}$ is the conduction heat flow inside the pipe.

- $\dot{Q}_{\text {convection,HTF }}$ is the conduction-convection heat flow from the internal surface of the pipe to the HTF. The model used of the heat transfer coefficient $\left(h_{\mathrm{HTF}}\right)$ is based on the Dittus-Boelter correlation (Cengel 2014) with $n=0.4$ :

$$
\begin{aligned}
& h_{\mathrm{HTF}}=\frac{\mathrm{Nu} \cdot k_{\mathrm{HTF}}}{2 \cdot r_{0}}, \\
& \mathrm{Nu}=0.023 \cdot \mathrm{Re}^{0.28} \cdot \mathrm{Pr}^{0.4}, \\
& \mathrm{Re}=\frac{\mathrm{v}_{\mathrm{HTF}} \cdot 2 \cdot r_{0} \cdot \rho_{\mathrm{HTF}}}{\mu_{\mathrm{HTF}}} \\
& \operatorname{Pr}=\frac{c_{p, \mathrm{HTF}} \cdot \mu_{\mathrm{HTF}}}{k_{\mathrm{HTF}}}
\end{aligned}
$$

where $\mathrm{Nu}, \mathrm{Re}$ and $\mathrm{Pr}$ are respectively the Nusselt, Reynolds and Prandtl numbers; $k_{\mathrm{HTF}}$ is the themal conductivity of the HTF, $r_{0}$ the internal radius of the absorber pipe, $v_{\mathrm{HTF}}$ is the mean HTF velocity, $\rho_{\mathrm{HTF}}$ is the density, $c_{p, \mathrm{HTF}}$ is the specific heat, and $\mu_{\mathrm{HTF}}$ represents the dynamic viscosity.

- $\dot{Q}_{\text {convection,lost }}$ is the heat flow rate lost by convection to the environment. This flow depends on the environment temperature and the wind velocity close to the PTC, based on the correlation corresponding to the heat transfer coefficient defined by Churchill $\mathrm{y}$ Bernstein in (Cengel 2014):

$$
\begin{aligned}
& h_{\text {air }}=\frac{\mathrm{Nu} \cdot k_{\text {air }}}{2 \cdot r_{1}} \\
& \mathrm{Nu}=0.3+\frac{0.62 \cdot \operatorname{Re}^{\frac{1}{2}} \cdot \operatorname{Pr}^{\frac{1}{3}}}{\left[1+\left(\frac{0.4}{\mathrm{Pr}}\right)^{\frac{2}{3}}\right]^{\frac{1}{4}}}\left[1+\left(\frac{R e}{282000}\right)^{\frac{5}{8}}\right]^{\frac{4}{5}} \\
& \operatorname{Re}=\frac{\mathrm{v}_{\text {wind }} \cdot 2 \cdot r_{1} \cdot \rho_{\text {air }}}{\mu_{\text {air }}} \\
& \operatorname{Pr}=\frac{c_{p, \text { air }} \cdot \mu_{\text {air }}}{k_{\text {air }}}
\end{aligned}
$$

where all the variables have the same meaning as above and are evaluated for the air medium at ambient temperature $T_{a m b}$. In particular, $v_{\text {wind }}$ is the wind velocity close to the outlet radius of the glass envelope $r_{1}$.

- $\dot{Q}_{\text {radiation,steel-lost }}$ is the radiation heat flow lost to the environment passing through the envelope glass. $\dot{Q}_{\text {radiation,glass-lost }}$ is the radiation heat flow lost to 
the environment. For both heat flows the sky temperature approximation considered is the following (Zarza 2003)

$$
T_{s k y}=T_{a m b}-10 .
$$

\section{MSL implementation of the TCP- 100 thermal losses model}

The main objective in this section is to implement the model of the PTC reusing the classes of the MSL. For the thermal losses model we have instantiated mostly the Modelica. Thermal. HeatTransfer subpackage classes, according to the phenomena involved: conduction, convection, and radiation. Only the parameters and variables needed to define the heat transfer coefficients and/or the thermal conductances had to be added to our model. More details can be read in the MSL documentation (Fritzson 2014).

Figure 9 shows the thermal losses model obtained by properly instantiating and parameterizing the MSL components. The connector port_a (instance of HeatPort_a) is the point where the boundary condition representing the fluid temperature will be connected. In a complete model of a PTC, this temperature is usually a state variable or an algebraic variable computed from an equation of state for the HTF, and thus, it is assumed to be known. The flows in the model are all implemented with the classes Modelica.Thermal.HeatTransfer . Components. The energy conservation in the glass envelope and the metal pipe masses are represented by the class HeatCapacitor. In the cases where the thermal conductances in the conduction-convection and radiation flow models were variables instead of parameters, RealExpression blocks have been properly used to consider this. In order to define boundary conditions, the wind velocity close to the absorber pipe, and the ambient temperature are assumed to be known from experimental measurements.

The relation of the instances of MSL classes presented in Figure 9, that implement the first principles models of the heat flows (detailed in section 3) is as follows: $\dot{Q}_{\text {absorbed,glass }}$ in Q_incident_glass; $\dot{Q}_{\text {absorbed,steel }}$ in Q_steel_incident; $\dot{Q}_{\text {conduction,glass }}$ in Q_Conduction_glass1 and Q_Conduction_glass2; $\dot{Q}_{\text {radiation,glass-steel }}$ in Q_radiation_steel_glass; $\dot{Q}_{\text {conduction,steel }}$ in Q_conduction_steel1 and Q_conduction_steel2; and, $\dot{Q}_{\text {convection,HTF }}$ is the convection heat flow from the pipe to the HTF, computed by Equation 1, considering the temperature difference between the boundary of the fluid port_a.T and the bulk fluid temperature (not represented in Figure 9).

The parts of the model not implemented in the components of the MSL have been implemented in Modelica code. For example, this is the case of all the variables and parameters associated with equations (1) to (3).

\section{Simulation results of the TCP-100 thermal losses model}

In this section we present the results of a simulation experiment of the Modelica losses model in Figure 9, performed with the Dymola tool.

In Figure 6 the boundary conditions applied to the model are shown: concentrated direct normal irradiance, ambient temperature, and wind velocity profile assumed close to the absorber pipe. In this case, the dynamics between the direct normal irradiance and the inlet HTF temperature have been included in order to illustrate the use of an experimental irradiance register from Plataforma Solar de Almería.
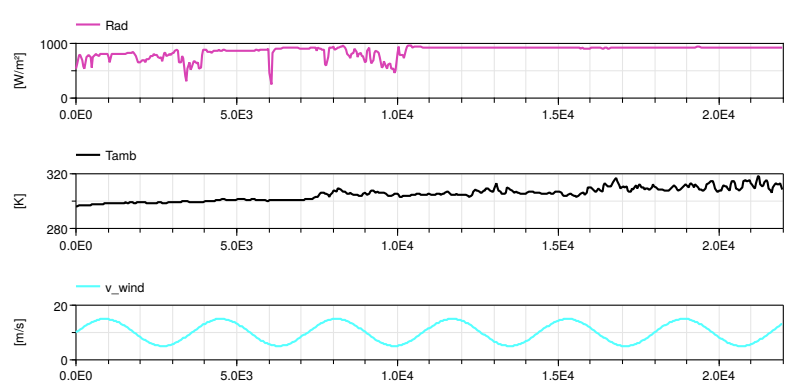

Figure 6. Boundary conditions in the simulation experiment. Direct normal solar irradiance (Rad), ambient temperature (T_amb), and wind velocity (v_wind).

In Figure 7, a comparison of the results of three different global losses model for the TCP-100 PTC is depicted. Q_corr presents the results of the model used in (Pérez et al. 2018) that is a global model assuming losses by convection and radiation from the glass envelope; Q_Pipe-maker presents the results obtained from a model based on the data specifications from the manufacturer of the TCP-100 PTC, and Q_Fpm shows the results of the first principles model presented in this article, as detailed in sections 3 and 4. In all three cases, the PTC model (excluding the thermal losses part) is the one presented in (Pérez et al. 2018). Although the simulation experiment shown in this figure contains nominal parameters for the model in Figure 9, calibration activities with experimental data in the TCP-100 facility might show some variations. It can be observed that the losses predicted by the model presented in this paper (Q_Fpm) are higher than those predicted by the manufacturer of the PTC (Q_Pipe-maker), and also higher than those predicted by the thermal model used in (Pérez et al. 2018). In the absence of the required experimental validation, our interpretation of this preliminary result is that the dependence of the wind velocity in the new proposed model could be producing higher thermal losses.

Figure 8 focuses on the presented first principles losses model, showing the different heat flow rate components under the boundary conditions profile provided in Figure 6. It is shown that the convection heat flow rate from the glass envelope (Qconv_lost) is the component with 


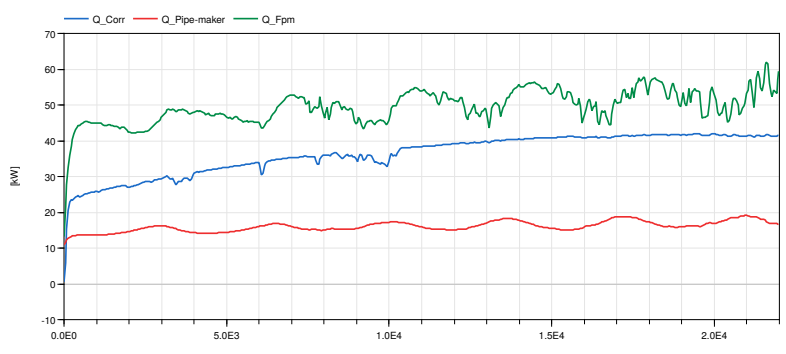

Figure 7. Simulation results of different thermal losses models: Q_corr presents the results of the model in (Pérez et al. 2018), Q_Pipe-maker presents the results obtained with a model based on the data specifications from the manufacturer of the TCP-100 PTC; and Q_Fpm represents the results of the first principles model presented in this article.

higher losses. The radiation losses from the steel pipe (Qrad_steel_lost) are lower than the convection losses from the glass envelope (Qconv_lost ); also, the component with the lowest losses is Qrad_glass_lost, from the glass envelope, due to the lower mean temperature of this part.

The presented results are considered preliminary because the parameters used for the simulation of the proposed model have been obtained from data specifications from the PTC manufacturer and the bibliography. To validate these preliminary results, a set of experiments will be performed in the TCP-100 facility, with the objective of calibrating the parameters according to the experimental data to be obtained in future tests.

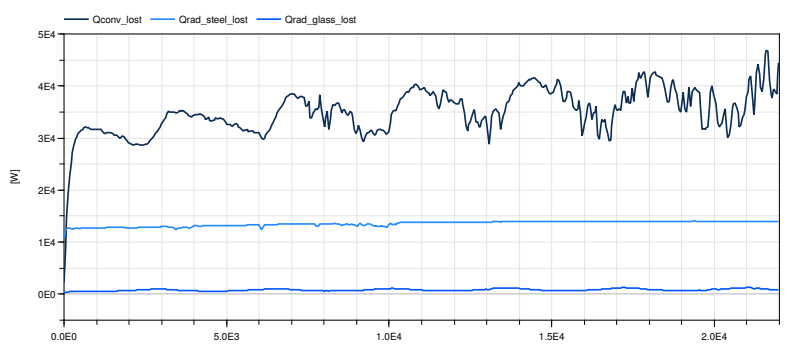

Figure 8. Simulation of the heat flow rates of thermal losses to the environment. Qconv_lost is the convection heat flow rate from the glass envelope, Qrad_steel_lost is the radiation heat flow rate from the steel pipe, and Qrad_glass_lost is the radiation heat flow rate from the glass envelope.

\section{Conclusions}

In this paper we have presented a thermal losses model of each of the PTCs in the TCP-100 solar themal power plant installed at Plataforma Solar de Almería (CIEMAT). This new model is based on first principles and includes an additional input variable with respect to the traditional approaches. The model is implemented with classes from the Modelica Standard Library (MSL), which have been complemented with the necessary equations to define the parameters and the variables required by the MSL, which have been obtained from diverse theoretical references.
This is an ongoing work and the results presented are only preliminary, and they need to be validated with experimental data to be obtained in the TCP-100 facility.

Future works might include the results of the calibration of parameters in the presented first principles model, based on the experimental data from the TCP-100 research facility.

\section{Acknowledgements}

The work of Luis J. Yebra was supported by the Universidad de Alcalá, through the Programa propio Giner de los Ríos Research Grant.

\section{References}

Bejan, Adrian (2016). Advanced engineering thermodynamics. John Wiley \& Sons.

Cengel, Yunus (2014). Heat and mass transfer: fundamentals and applications. McGraw-Hill Higher Education.

Fritzson, Peter (2014). Principles of object-oriented modeling and simulation with Modelica 3.3: a cyber-physical approach. John Wiley \& Sons.

Gallego, Antonio J., Luis J. Yebra, and Eduardo F. Camacho (2018). "Gain Scheduling Model Predictive Control of the New TCP-100 Parabolic Trough Field". In: IFACPapersOnLine 51.2, pp. 475-480. ISSN: 24058963. DOI: 10. 1016/j.ifacol.2018.03.080. URL: https://doi.org/10.1016/j. ifacol.2018.03.080.

Gallego, Antonio J., Luis J. Yebra, Eduardo F. Camacho, and Adolfo J. Sánchez (2016). "Mathematical Modeling of the Parabolic Trough Collector Field of the TCP-100 Research Plant". In: Proceedings of The 9th EUROSIM Congress on Modelling and Simulation, EUROSIM 2016, The 57th SIMS Conference on Simulation and Modelling SIMS 2016, pp. 912-917. DOI: 10.3384/ecp17142. URL: https://doi.org/ $10.3384 /$ ecp 17142 .

Márquez, Francisco M., Pedro J. Zufiria, and Luis J. Yebra (2020). "Port-Hamiltonian modeling of multiphysics systems and object-oriented implementation with the Modelica language". In: IEEE Access 8, pp. 105980-105996. DOI: 10. 1109/access . 2020.3000129. URL: https://ieeexplore.iee. org/document/9110502.

Pérez, J et al. (2018). "First Principles System Level Modelling of TCP-100 Facility for Simulation of Operation Modes". In: IFAC-PapersOnLine 51.2, pp. 481-486. DOI: 10.1016/ j.ifacol.2018.03.081. URL: https://doi.org/10.1016/j.ifacol. 2018.03.081.

Wagner, Michael J. and Paul Gilman (2011). Technical manual for the SAM physical trough model. Vol. 303. June, pp. 2753000. URL: http://www.nrel.gov/docs/fy11osti/51825.pdf.

Yebra, Luis J., Francisco M. Márquez, and Pedro J. Zufiria (2020). "Simulation of TCP-100 Facility System Level Model for Operation Training Purposes". In: Proceedings of The 7th Annual Conf. on Computational Science \& Computational Intelligence (CSCI'20). DOI: 10.1109/CSCI51800. 2020.00251.

Zarza, Eduardo (2003). "Generación directa de vapor con colectores solares cilindro parabólicos Proyecto DIrect Solar Steam (DISS)". PhD thesis. Universidad de Sevilla, pp. 1480. URL: https://idus.us.es/handle/11441/15300. 


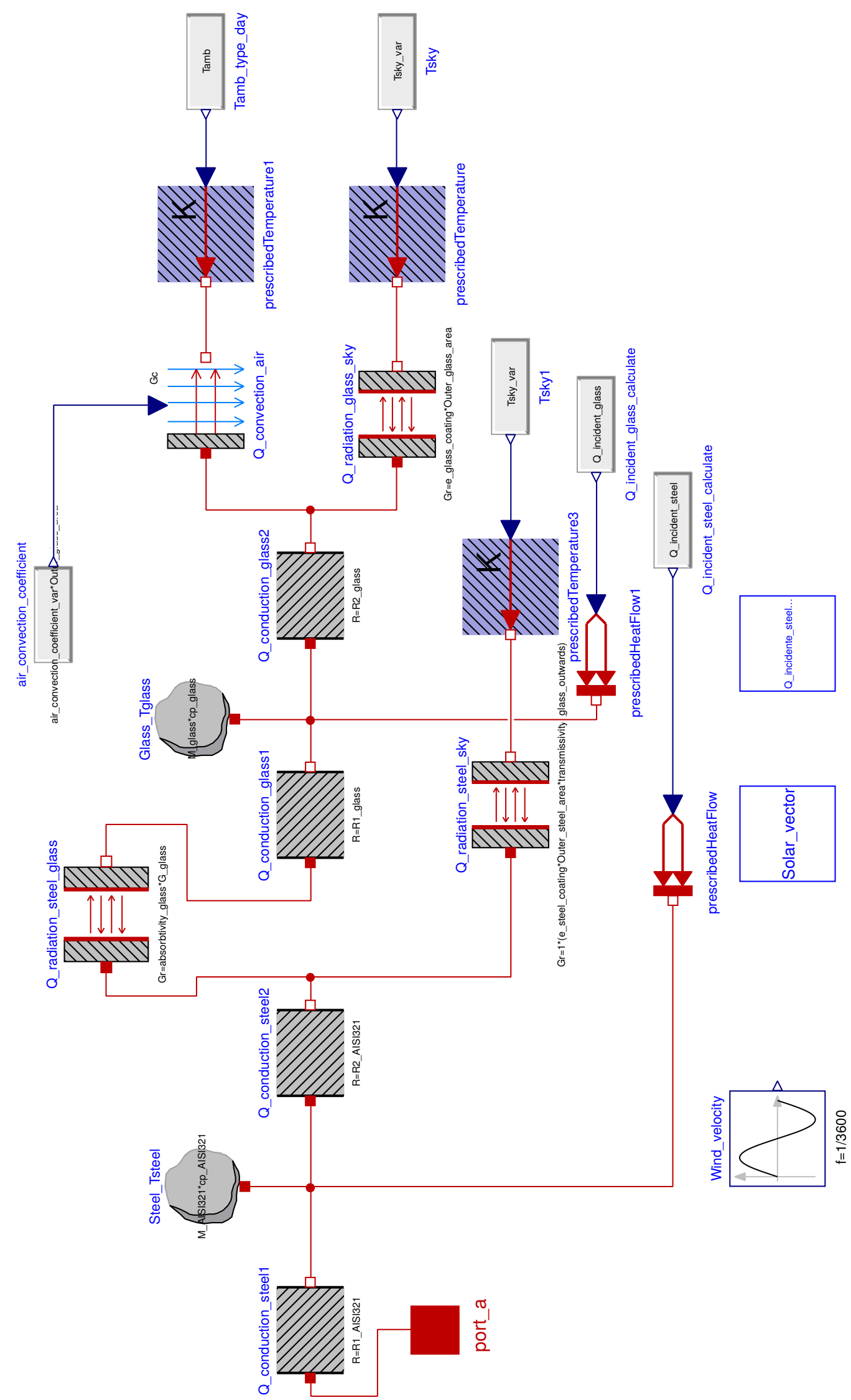

Figure 9. Modelica model of the thermal losses of the PTC TCP-100 implemented with the Modelica Standard Library. 